\title{
Model-Based Multi-Object Segmentation via Distribution Matching
}

\author{
Paper \#198
}

\begin{abstract}
A new algorithm for the segmentation of $3 D$ deformable objects from $3 D$ images is presented. This algorithm relies on learned shape and appearance models for the objects of interest. The main innovation over similar approaches is that there is no need to compute a pixelwise correspondence between the model and the image; instead, probability distributions are compared. This allows for a faster, more principled algorithm. Results of the algorithm are shown both on synthetic images and for the segmentation of the prostate, bladder, and rectum from medical images.
\end{abstract}

Keywords: deformable segmentation, prostate segmentation, shape and appearance model, medical image segmentation.

\section{Introduction}

The segmentation of 3D deformable objects is both an important and interesting problem in computer vision. It is important because of its natural application in the medical arena; for example, segmentation of tumors from CT or MRI images can be critical in the treatment of cancer. On the other hand, it is interesting because of the algorithmic challenges inherent in extracting deformable objects from real-world 3D images. In the context of medical imagery, the key segmentation-related challenges are the following:

- Challenge \#1: The objects of interest are often diffuse and lack strong edges.

- Challenge \#2: There are often many objects, both of interest and not of interest, within a small volume.

- Challenge \#3: Many objects have fairly similar intensity profiles. Typically, this effect cannot be removed by simple pre-processing such as histogram equalization.

- Challenge \#4: Many of the objects are of roughly the same shape. For example, the prostate and bladder are both "somewhat deformed" spheres.

The algorithm presented in this paper uses learned models for both the shape and appearance of objects to achieve segmentation; learning both types of information is the only reasonable way to deal with all 4 challenges. Our algorithm is certainly not the first algorithm to combine shape and appearance. However, existing algorithms which use both shape and appearance models (such as [4]) require a pixelwise correspondence between the model and the image; this correspondence is often very difficult to compute, and can be extremely time-consuming. Instead, our algorithm characterizes a model object by (a) its shape and (b) a probability distribution of the intensities (or colours, textures) of the pixels within its interior. As a result, comparing a particular model object to the image is as simple as comparing two probability distributions. The algorithm allows the shape to evolve until the optimal match is found.

The remainder of the paper is organized as follows. Section 2 reviews the existing literature on segmentation of 3D deformable objects. Section 3 is the heart of the paper; it derives the equations which comprise the segmentation algorithm. Section 4 presents results on a synthetic image, to illustrate the advantages of this type of approach. Section 5 demonstrates the algorithm's effectiveness in a medical application: segmentation of the prostate, as well as its surrounding structures (bladder, anterior rectal wall) from medical imagery. Finally, Section 6 concludes.

\section{Prior Work on Segmentation of 3D Deformable Objects}

Existing algorithms can be categorized based on the type of learned information they use.

No learned information. The main exemplar of this type of approach is the traditional active contour or "snake" [8]. More recent work (e.g. [1]) has focused on geometric curve evolution, combined with level sets [11], to allow for both topological changes to the object, as well as greater numerical stability. Standard active contour methods that seek edges tend to have difficulty when the objects to be segmented are blurry or not sharply delineated from the background (such as the prostate in Section 5 below). Extensions such as [3] try to segment on the basis of appearance by evolving the surface based on simple criteria related to the intensities (or colors) of the pixels in its interior; these methods achieve greater accuracy and robustness at the cost of a major reduction in speed. In general, algorithms which do not use learned information are constrained in terms of what they can segment; they will have difficulties with each 
of the four challenges posed above.

Learned shape models. Some researchers have augmented a level-set active contour segmentation algorithm with a PCA term that biases the curve evolution towards shapes that are judged to be more likely based on the training set $[10,15]$. These methods have been extended to allow for simple (non-learned) models of appearance [19, 20]; for example, the intensities within the segmented areas may be forced to have highly differing means or variances. Segmentation of 3-D medical images has also been accomplished by the coarse-to-fine deformation of a shape-based medial representation model, or "m-rep" [13, 17]. Algorithms which possess only a learned shape model will typically fall prey to challenges 3 and 4 .

Learned appearance models. One version of this type of segmentation involves the non-parametric warping of a target surface to a deformable atlas [7]. Contours from the atlas can then be transferred onto the target volume. This type of method will have trouble with challenges 2, 3 , and 4 . In the absence of some physiological interpretation of a given mapping or incorporation of training data, this method can assign a low cost to seemingly-plausible mappings and warpings that would never occur in practice. Speed is a drawback as well, since a warping between two entire image volumes is generally computed. Some active contour models [12] assume that one has a probabilistic characterization of appearance which is learned beforehand.

Learned shape and appearance models. There are a variety of methods that model the shape and appearance of an object using principal component analysis (PCA). The standard-bearer for such methods is the "active shape and appearance model" of Cootes et al. [4], which has been successfully applied to the three-dimensional segmentation of medical volumes, including magnetic resonance images of the brain, heart, and articular cartilage $[6,9,22]$.

The main drawback of active shape and appearance model has already been mentioned in Section 1; they require the computation of a pixelwise correspondence between the model and the image. We will say more about this in Section 3.2.

\section{The Segmentation Algorithm}

In this section, we describe the heart of the algorithm: the procedure for fitting a combined shape-appearance model to an image. This optimal fitting of the model results in the segmentation of the image. The basic idea is as follows. The shape is given by a description of the surface, or multiple surfaces in the case of multi-object segmentation. The appearance is described by a probability distribution of some photometric variable inside the object of interest, or multiple distributions in the case of multi-object segmentation. A shape-appearance pair is then given by (surface, distribution), and this pair is considered sufficient to characterize an object for the sake of segmentation. The learned model is a low-dimensional manifold in the space of such pairs. To verify how well any particular shape-appearance pair matches the image, we compute the empirical distribution of the photometric variable inside the shape within the image; this distribution is then compared to the appearance model. We therefore evolve the shape of an object (or multiple objects) until the empirical distribution(s) best matches the model distribution(s). In the remainder of this section, we flesh out these ideas.

\subsection{Terminology}

In this section, we describe some of the notation needed to define the problem rigorously. In the case of single-object segmentation, a model-instance is described by a (surface, distribution) pair. The distribution is taken over some photometric variable; in the experiments we perform, this variable is grayscale intensity, however, it may also be colour or texture. Given that the image is discrete-valued, we will assume a probability mass function over the intensity. However, all of the analysis below can easily be transferred to the case of probability density functions (which might be more relevant in the case of textures). In the case of multiobject segmentation, a model-instance will be specified by $J$ (surface, distribution) pairs, one for each object.

We assume each surface is a topological sphere, and may therefore be written $S: \mathcal{S}^{2} \rightarrow \mathbb{R}^{3}$. For convenience, we will denote a point on the surface using a parametrization of $\mathcal{S}^{2}$ as $S(u)$; however, the particular parametrization chosen is unimportant. Let us denote the image by $I: \mathbb{R}^{3} \rightarrow\{1, \ldots, n\}$; the image is piecewise constant, where the "pieces" correspond to voxels (which have positive volume). We denote the probability distribution by $\mathbf{q}=\left(q_{1}, \ldots, q_{n}\right)$, where $q_{i}=\operatorname{prob}(I(x)=i)$; of course $q_{i} \geq 0$ and $\sum_{i} q_{i}=1$. Thus, a model-instance is given by $(S(\cdot), \mathbf{q})$. The shape-appearance model is a lowdimensional manifold in the space of such model-instances; a $d$-dimensional model is parametrized by $\beta \in \mathbb{R}^{d}$, and we will write $(S(\cdot ; \beta), \mathbf{q}(\beta))$ (sometimes condensing $(S(\cdot ; \beta)$ to $S(\beta)$ ). Particular forms for the shape and appearance model $(S(\beta), \mathbf{q}(\beta))$ are discussed in Sections ?? and 5; in the subsequent derivation, the particular form is unimportant.

The goal is to find the particular model-instance, i.e. the particular $\beta$, for which the model best matches the image. In the following section, we describe a natural criterion for scoring such matches.

\subsection{Segmentation Criterion}

Given a surface $S$, let $\mathbf{p}^{S}$ be the distribution (probability mass function) of intensities lying inside the surface $S$. This 
can be formally defined as follows. Let $V$ be the volume inside of $S$; that is, let $S=\partial V$. In this case,

$$
p_{i}^{S}=\frac{\int_{x \in V} \delta(I(x), i) d x}{\int_{x \in V} d x}
$$

where $\delta(i, j)=1$ if $i=j$ and 0 otherwise. We will refer to $\mathbf{p}^{S}$ as the empirical distribution corresponding the surface $S$.

The goal of segmentation is to find a region in the image which is most like the model. That is, we would like to find a model shape $S(\beta)$ whose empirical distribution $\mathbf{p}^{S(\beta)}$ most closely matches its model distribution $\mathbf{q}(\beta)$. In other words, the segmentation can be posed as

$$
\min _{\beta} K\left(\mathbf{p}^{S(\beta)}, \mathbf{q}(\beta)\right)
$$

where $K$ is some suitable measure of dissimilarity between probability distributions. There are several obvious candidates for $K$ from information theory. We choose the Kullback-Leibler divergence,

$$
K(\mathbf{p}, \mathbf{q})=\sum_{i=1}^{n} p_{i} \log \frac{p_{i}}{q_{i}}
$$

Note a key feature of this segmentation algorithm: unlike other joint shape-appearance model-based algorithms, there is no need to find a correspondence between the pixels of the model and those of the image. For example, in [4] finding the correspondence involves repeatedly computing Delaunay Triangulations in order to warp the image; this is by far the most time-consuming and least rigorous part of the entire algorithm. In our case, by contrast, pixels are not compared directly; instead, distributions are compared. While some information is obviously lost in performing distribution comparisons instead of pixel-wise comparisons, we show that in relevant experiments (for both synthetic images and real medical images) this loss of information does not adversely affect performance. Of course, distribution comparison measures may be computed considerably faster than pixelwise correspondences.

When we wish to segment multiple objects at once, our model is given by $J$ object descriptors $\left\{\left(S_{j}(\cdot ; \beta), \mathbf{q}_{j}(\beta)\right)\right\}_{j=1}^{J}$, and the goal is then to solve

$$
\min _{\beta} \sum_{j=1}^{J} K\left(\mathbf{p}^{S_{j}(\beta)}, \mathbf{q}_{j}(\beta)\right)
$$

Note that there is a single parameter vector $\beta$ which controls all of the objects; this captures the notion that the objects' shapes and appearances may be interrelated. Although a more general version of this criterion might be a weighted sum of Kullback-Leibler divergences, we have found the unweighted criterion works well in practice.

\subsection{Optimization of the Criterion}

We wish to minimize

$$
K(\beta) \equiv K\left(\mathbf{p}^{S(\beta)}, \mathbf{q}(\beta)\right)
$$

in the case of single object segmentation. (We will only deal with the single object case in this section; the multi-object case follows straightforwardly.) We will solve for a local minimum of the criterion via gradient descent, i.e.

$$
\frac{d \beta}{d t}=-\frac{\partial K}{\partial \beta}
$$

The computation of the derivative of $K$ with respect to the parameters $\beta$ is complicated by the reliance of the surface itself on the parameters.

From equation (2), we have that

$$
\frac{\partial K}{\partial \beta}=\sum_{i=1}^{n}\left[\left(1+\log \frac{p_{i}}{q_{i}}\right) \frac{\partial p_{i}}{\partial \beta}-\frac{p_{i}}{q_{i}} \frac{\partial q_{i}}{\partial \beta}\right]
$$

where we have shortened $p_{i}^{S(\beta)}$ to $p_{i}$ and $q_{i}(\beta)$ to $q_{i}$. But equation (1) yields

$$
p_{i}^{S(\beta)}=\frac{\int_{x \in V(\beta)} \delta(I(x), i) d x}{\int_{x \in V(\beta)} d x} \equiv \frac{N_{i}^{S(\beta)}}{V(\beta)}
$$

so that

$$
\frac{\partial p_{i}}{\partial \beta}=\frac{1}{V}\left(\frac{\partial N_{i}}{\partial \beta}-p_{i} \frac{\partial V}{\partial \beta}\right)
$$

In order to compute $\partial N_{i} / \partial \beta$ and $\partial V / \partial \beta$, we need to be able to determine derivatives of the form $\partial \psi / \partial \beta$, where $\psi=\int_{x \in V(\beta)} \nu(x) d x$. The variational derivative of $\psi$ with respect to the surface $S$ (where $S=\partial V$ ) is given by $\frac{\delta \psi}{\delta S}=$ $\nu(u) n(u)$, where $n(u)$ is the normal to the surface at the point $S(u)$ (see, for example, [2]). It can be then be shown by a sort of generalized chain rule that

$$
\frac{\partial \psi}{\partial \beta}=\int_{u \in \mathcal{S}^{2}} \nu(u) \frac{\partial S}{\partial \beta}(u ; \beta) n(u ; \beta) d u
$$

where $\partial S / \partial \beta$ is a $d \times 3$ matrix $(d=\operatorname{dim}(\beta))$. To simplify future computations, we introduce the notation

$$
\Gamma(u ; \beta)=\frac{1}{V(\beta)} \frac{\partial S}{\partial \beta}(u ; \beta) n(u ; \beta)
$$

so that

$$
\frac{\partial \psi}{\partial \beta}=\int_{u \in \mathcal{S}^{2}} V(\beta) \Gamma(u ; \beta) \nu(u) d u
$$

We have, therefore, that

$$
\begin{aligned}
\frac{1}{V} \frac{\partial N_{i}}{\partial \beta} & =\int_{u \in \mathcal{S}^{2}} \Gamma(u ; \beta) \delta(I(S(u ; \beta)), i) d u \\
\frac{1}{V} \frac{\partial V}{\partial \beta} & =\int_{u \in \mathcal{S}^{2}} \Gamma(u ; \beta) d u
\end{aligned}
$$



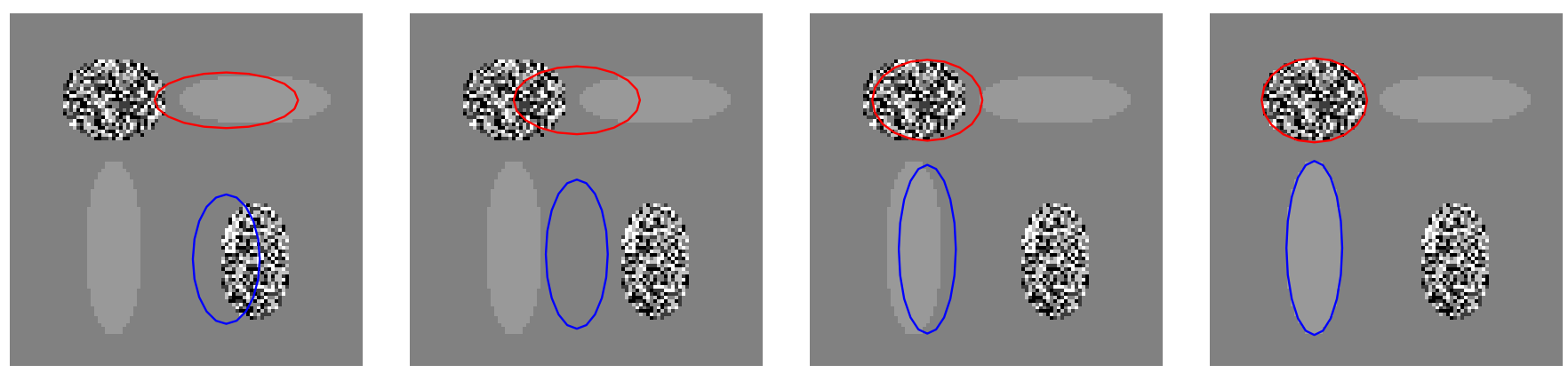

Figure 1: Tracking a synthetic sequence. (a) Initial position; (b), (c) intermediate positions; (d) final position.

Some simplification gives

$$
\begin{aligned}
\frac{\partial K}{\partial \beta} & =\int_{\mathcal{S}^{2}} \Gamma(u ; \beta)\left[\sum_{i=1}^{n} \delta(I(S(u ; \beta)), i)\left(1+\log \frac{p_{i}}{q_{i}}\right)\right] d u \\
& -\left(\int_{\mathcal{S}^{2}} \Gamma(u ; \beta) d u\right)\left[\sum_{i=1}^{n} p_{i}+p_{i} \log \frac{p_{i}}{q_{i}}\right]-\sum_{i=1}^{n} \frac{p_{i}}{q_{i}} \frac{\partial q_{i}}{\partial \beta} \\
& =\int_{\mathcal{S}^{2}} \Gamma(u ; \beta) d u+\int_{\mathcal{S}^{2}} \Gamma(u ; \beta) \log \frac{p_{I(S(u ; \beta))}}{q_{I(S(u ; \beta))}} d u \\
& -\int_{\mathcal{S}^{2}} \Gamma(u ; \beta) d u-K(\beta) \int_{\mathcal{S}^{2}} \Gamma(u ; \beta) d u-\sum_{i=1}^{n} \frac{p_{i}}{q_{i}} \frac{\partial q_{i}}{\partial \beta}
\end{aligned}
$$

which finally yields

$\frac{\partial K}{\partial \beta}=\int_{\mathcal{S}^{2}} \Gamma(u ; \beta)\left(\log \frac{p_{I(S(u ; \beta))}}{q_{I(S(u ; \beta))}}-K(\beta)\right) d u-\sum_{i=1}^{n} \frac{p_{i}}{q_{i}} \frac{\partial q_{i}}{\partial \beta}$

We will use equation (4) to find a minimum of the Kullback-Leibler divergence with respect to the model parameters, via gradient descent. In the next section, we discuss some implementation issues that arise in the computation of $\partial K / \partial \beta$.

\subsection{Implementation Issues}

In general, we cannot compute the integral in equation (4) analytically; we must resort to a finite element method. This is relatively straightforward, given that the surface representation we use is that of a mesh (simplicial complex). For any triangle of the mesh, the normal is fixed; furthermore, the triangles are chosen to be small enough so that neither $\partial S / \partial \beta$ nor $I$ varies much over the triangle. As a result, we can approximate the integral in equation (4) by

$$
\sum_{t \in T} \Gamma\left(u_{t} ; \beta\right)\left(\log \frac{p_{I\left(S\left(u_{t} ; \beta\right)\right)}}{q_{I\left(S\left(u_{t} ; \beta\right)\right)}}-K(\beta)\right) a_{t}
$$

where $T$ is the set of triangles in the mesh, $u_{t}$ is a representative point on the triangle $t$ (typically the centroid), and $a_{t}$ is the area of the triangle $t$.

\section{Results on Synthetic Data}

In this section, we demonstrate some attributes of the algorithm on synthetic data. There are two objects of interest, each of which are ellipsoids; the shape model allows for the ellipsoids to translate and deform. The appearance model is chosen to be fixed; the upper ellipsoid has a near-uniform distribution, allowing for a range of grayscale intensities, while the lower ellipsoid has a delta-like distribution, allowing for only one grayscale intensity. Figure 1 shows one slice of the 3D image, which is $100 \times 100 \times 40$; in this image, there are 4 ellipsoids, of which the true pair are on the left of the image (note that the upper one has a uniform distribution, whereas the lower one has a delta-like distribution). The extra two ellipsoids are inserted to simulate challenges 2, 3, and 4 mentioned in Section 1; that is, there are many objects within the area of interest with similar shapes and intensity profiles.

The results are displayed in Figure 1. Note that while the initial position is very close to the wrong pair of ellipsoids, the algorithm translates and deforms the ellipsoids according to the shape model until they coincide exactly with the true pair. All that is needed is a slight overlap of the initial position of the upper ellipsoid with the true object; this allows it to "sense" the true object, and to note that its empirical distribution may be made closer to the model distribution by translating leftward (and ultimately deforming as well). On an uncompiled MATLAB implementation running on a $1.67 \mathrm{GHz}$ AMD machine with $448 \mathrm{MB}$ RAM, the algorithm takes 21 seconds to run.

Let us contrast this performance with the performance which would be provided by the three classes of algorithms which do not use both shape and appearance information (see Section 2). Algorithms which do not use any learned information, such as snakes, will identify all four objects; there is nothing to distinguish amongst them. Algorithms which rely only on learned shape models, such as [20], may identify either pair of ellipsoids, as both pairs are both instances of the multi-object shape model; again, on the basis of shape only, there is nothing to distinguish between the 
pairs. Algorithms which rely only on learned appearance models, such as [12], may either identify all of the objects, or one particular pair arbitrarily; on the basis of appearance only, there is no way of differentiating between the top left ellipsoid and the bottom right ellipsoid, as both have uniform distributions. Of course, the active shape and appearance techniques of [4] do not suffer from these drawbacks; however, as has been pointed out in Section 2, these methods require the computation of a pixelwise correpondence between the model and the image, which can be very timeconsuming.

This synthetic image was designed to point out the flaws which result from not using both learned shape and appearance models; while in some sense the image is contrived, it does reflect the underlying reality of medical imagery: that in such images, there are many objects within the area of interest with similar shapes and intensity profiles. We now turn to a real example of such imagery.

\section{Application: Prostate Radiotherapy}

In this section, we present the results of our segmentation algorithm applied to an important real-world problem in medical imaging: rapid, automatic contouring of the prostate and other structures from volumetric computed tomography (CT) datasets for the purpose of image-guided radiation therapy.
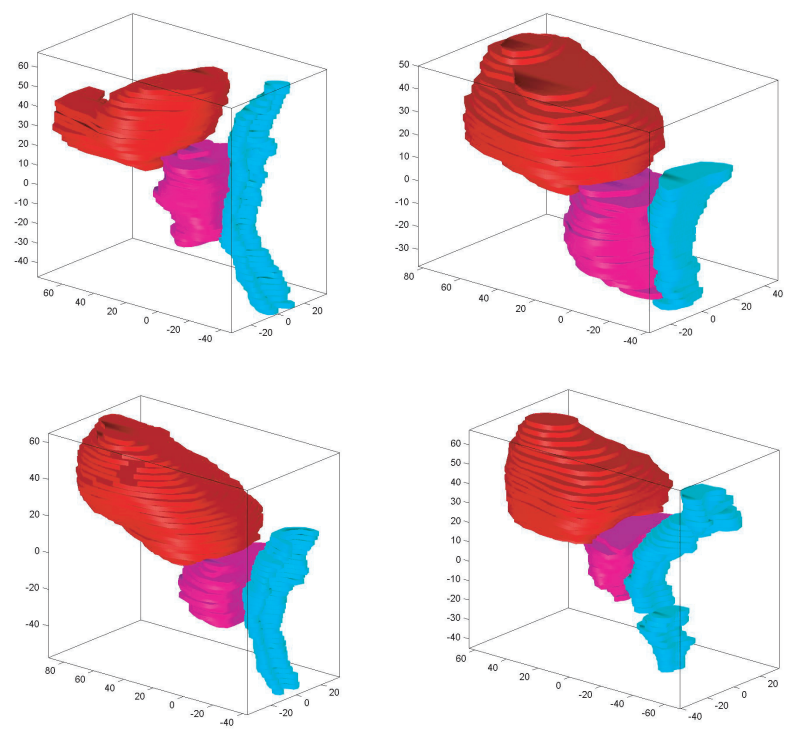

Figure 2: Hand-drawn bladder (red, left), prostate (pink, middle), and anterior rectal wall (blue, right) contours from four different patients that illustrate substantial inter-patient variability.

Prostate cancer is the second leading cause of cancer death for American men. An important advance in cancer treatment with radiation has been the introduction of a new technology known as intensity modulated radiotherapy (IMRT). This is a computer-controlled method of delivering radiation using several beams from different angles that can precisely irradiate a target that has a complex (e.g. nonconvex) 3-D shape while simultaneously avoiding normal radiation-sensitive tissues.

The bottleneck in 3D IMRT systems is the significant amount of time and human intervention required to delineate the prostate and nearby structures on each scan. Usually a radiation oncologist takes $30-45$ minutes to outline all of the structures of interest. Performing this operation each of the 30-40 times a patient is treated is burdensome. Using an uncompiled MATLAB implementation on a modest machine (1.67 GHz AMD machine with $448 \mathrm{MB}$ RAM), our algorithm performs the same procedure in five minutes.

The state of the art in computer vision algorithms applied to prostate segmentation is considerably less advanced than for other sites. In the best hospitals across the country, the status quo is still manual contouring. Several vision approaches have been presented for prostate segmentation from ultrasound; one of the most effective is an activecontour-based method proposed by Shen, Zhan and Davatzikos [16]. The work most comparable to the algorithm described here was recently proposed by Tsai et al. [19, 20] and applied to 3D MRI images of the prostate. These algorithms do not use learned appearance models; the disadvantage of using only learned shape models has already been discussed in Sections 2 and 4.

\subsection{Learning the model}

In order to implement equation (4), we must have a shapeappearance model, $(S(\beta), \mathbf{q}(\beta))$; the goal is to learn this model from training data. Our training set consisted of 21 sets of $512 \times 512 \times 90 \mathrm{CT}$ images of the male pelvis, in each of which a radiation physicist had outlined the prostate, bladder, and anterior rectal wall. We chose to segment the bladder and anterior rectal wall in addition to the prostate since they are radiation-sensitive structures that should be detected and avoided during radiation delivery. It turns out that the appearances (i.e. probability mass functions) of the three organs vary very little with shape; as a result, a fixed appearance model is sufficient. For each organ, the histogram is learned by simply averaging over the histograms in all of the training images.

The shape of the organs, on the other hand, does vary quite a bit; Figure 2 illustrates the physician-drawn contours of bladder, prostate, and anterior rectal wall surfaces from several patients. It is clear that there is a substantial amount of inter-patient variability in this data set. In the derivation of equation (4), no restrictions were placed on the geometry of the manifold representing the shape model $S(\beta)$. How- 
ever, in practice we use a linear representation derived from principal component analysis, a common technique in the model-based segmentation literature; see for example [4]. The general scheme is as follows: the surface is represented as a mesh, and is thus specified by a vector whose elements are the $x-, y$-, and $z$-coordinates of each of the vertices. The vectors representing the three organs are then stacked into a combined vector. There is one such combined vector for each training image, and PCA is performed on these combined vectors.

In this case, points from homologous structures were automatically extracted and put into correspondence using a variational-implicit-surface-based algorithm [21] that resamples every set of contours to have the same number of slices and the same number of points equally spaced around each contour. In this example, we resampled each object to have 20 slices with 20 points on each contour, and constructed a PCA model using the 1200 points from each patient. Figure 3 shows the effect of varying the two most dominant modes of the joint shape model. We can see that the model is able to capture nonrigid physiological variability (such as variable bladder filling) automatically.

Naturally, as more modes are used in a PCA model, it can potentially fit a new image with higher accuracy. On the other hand, using more modes involves a higherdimensional search space with more opportunities for numerical problems. Based on the modes-vs.-error characteristics of our joint model, we chose to use 15 modes in the shape model.

\subsection{Results}

We initialized the model using $\beta=0$ in equation (3), that is, at the position of the mean shape, and allowed it to converge. Note that this is in contrast to other algorithms where rough manual placement is required to guarantee convergence to the correct result. The results for one of the 21 patients is shown in Figure 4. The model and segmentation algorithms are fully three-dimensional, but the results in Figure 4 are represented as 2-D slices for easy visualization. One can appreciate the difficulty of the segmentation problem in this context: the CT images have relatively low contrast in the area of interest, and the prostate, bladder, and anterior rectal wall have gray-scale intensities that are very similar to each other as well as to structures in many other regions. Figure 5 shows the final multi-object segmentation result and the ground truth as 3-D contours for comparison.

We can determine the best possible fitting result by projecting the known ground truth $G$ onto the 15-mode shape model to obtain a point set $M_{15}$ and computing the Hausdorff error between the two point sets, defined as

$$
H\left(G, M_{15}\right)=\max \left(\max _{x \in G} \min _{y \in M_{15}}\|x-y\|, \max _{y \in M_{15}} \min _{x \in G}\|x-y\|\right)
$$

We find that the best possible Hausdorff errors using our model are $6.1 \mathrm{~mm}, 5.5 \mathrm{~mm}$, and $8.9 \mathrm{~mm}$, for the bladder, prostate, and anterior rectal wall respectively. Of these, we are most concerned with the prostate since this is the target of the radiation. In contrast, the fully automatic segmentation results presented in the second row of Figure 4 represent Hausdorff errors of $9.8 \mathrm{~mm}, 6.1 \mathrm{~mm}$, and $9.4 \mathrm{~mm}$, for the bladder, prostate, and anterior rectal wall respectively. We are currently working with radiation physicists to define an error measure that is more relevant to the regions of interest for image-guided therapy.

17 iterations of a discrete version of equation (3) were required; the corresponding running time was 5 minutes and 7 seconds on a $1.67 \mathrm{GHz}$ AMD machine with $448 \mathrm{MB}$ RAM. Therefore, we conclude that our segmentation algorithm has substantial promise for the problem of rapid, automatic contouring.

\section{Conclusions and Future Work}

We have demonstrated a segmentation algorithm which matches a learned model of shape and appearance to an image by comparing empirical and model probability distributions. The algorithm produces good results on both synthetic and real medical images. Unfortunately, the algorithm relies on local optimization. Future work will focus on coarse-to-fine methods of sampling the model space, to ensure that the algorithm is not trapped in an incorrect optimum.

Another direction for future work relates to the model itself. Strict linear models can require many modes to capture complex variations, and are inappropriate for constrained quantities like histograms. We are investigating multilinear [5] or non-linear $[14,18]$ models to that will allow us to use fewer parameters, and will ensure that any model parameter values produce a "valid" instance of shape and appearance.

Furthermore, we will shortly obtain a corpus of training data that includes CT volumes and contours that vary along both inter-patient and intra-patient axes. That is, for each of many patients we will have many examples of that patient's bodily state. Using this data, we plan to learn a model of inter- and intra-patient variation that is more suitable for image-guided therapy applications than the inter-patient model described here. We expect intra-patient data to have much less inherent variability than inter-patient data, and hence for our segmentation algorithms to perform more accurately.

\section{References}

[1] V. Caselles, R. Kimmel, and G. Sapiro. On geodesic active contours. Int. J. Comput. Vis., 22(1):61-79, 1997. 

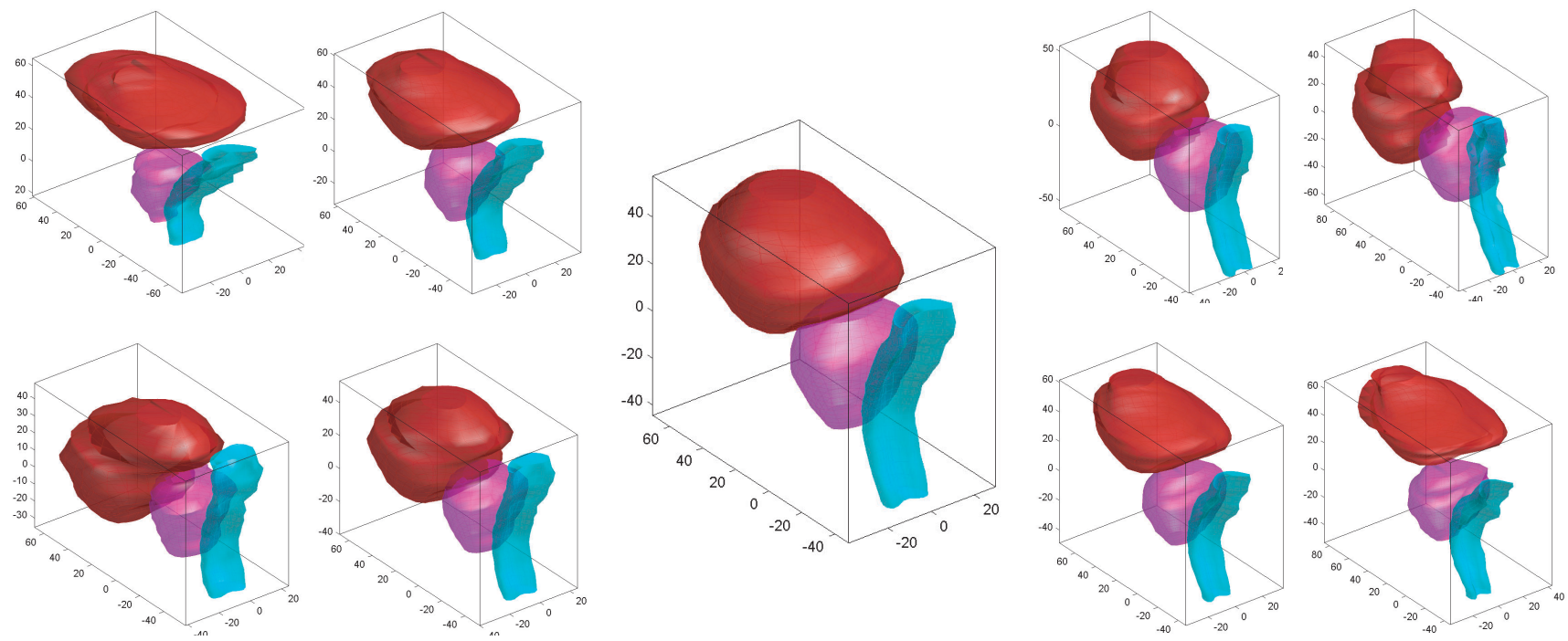

Figure 3: Center: The mean shape of the PCA model of the bladder, prostate, and rectum. The rows correspond to varying the two most dominant modes of the joint shape model of the bladder, prostate, and anterior rectal wall. The weights on the modes, from left to right, are $\left\{-2 \sqrt{\lambda_{i}},-\sqrt{\lambda_{i}}, 0, \sqrt{\lambda_{i}}, 2 \sqrt{\lambda_{i}}\right\}$, with $\sqrt{\lambda_{1}}=373$ and $\sqrt{\lambda_{2}}=289$.
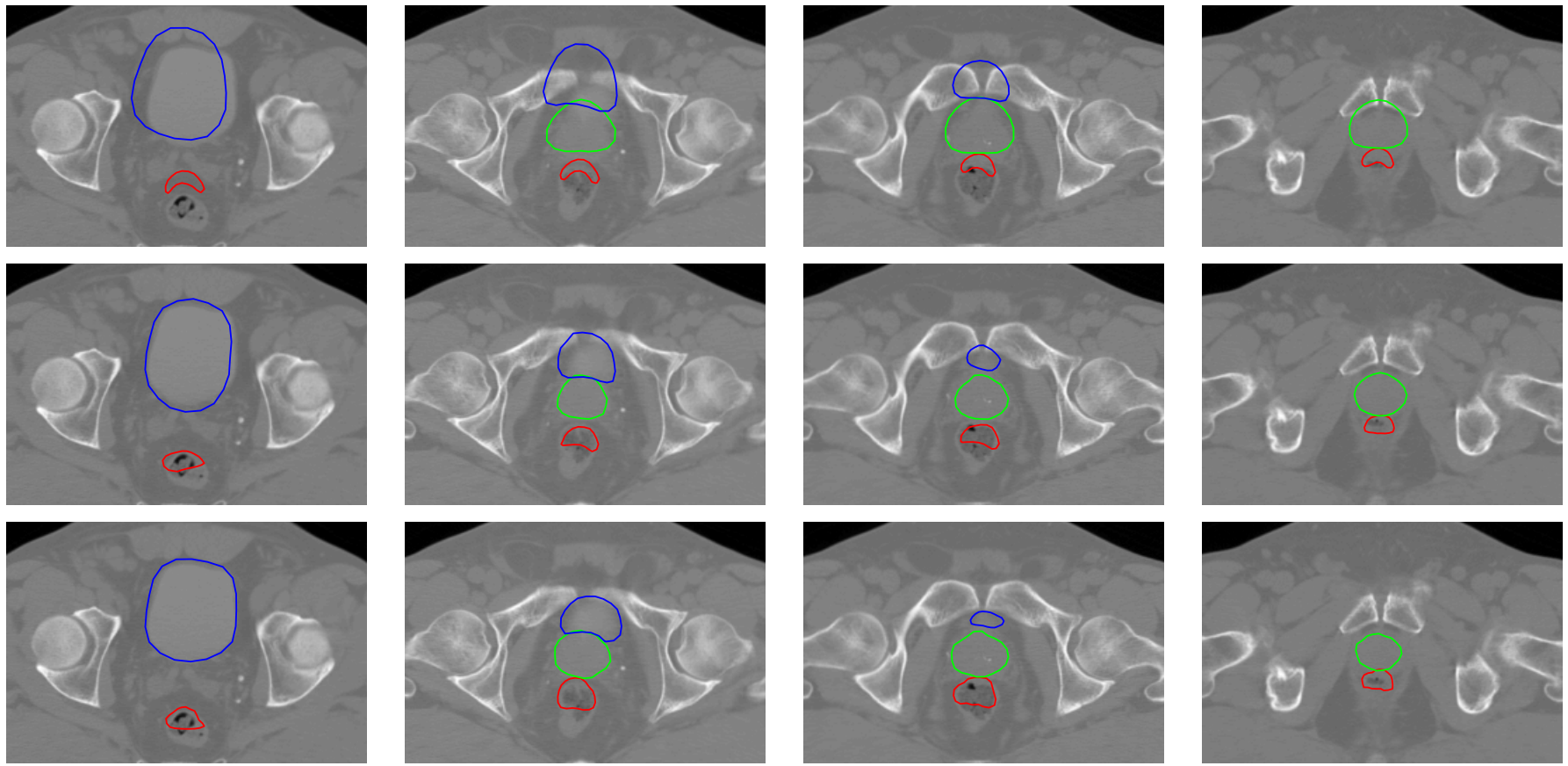

Figure 4: Segmentation results for slices 27, 34, 36, and 41 of patient 2664. The top row shows the initial boundaries for the segmentation (corresponding to the mean shape). The middle row shows the segmentation result at convergence. The bottom row shows the hand-drawn ground-truth contours supplied by a radiation physicist. In each image, the top (blue) contour is the bladder, the middle (green) contour is the prostate, and the bottom (red) contour is the anterior rectal wall. Note that the prostate is not visible in the first column, and the bladder is not visible in the last column.

[2] A. Chakraborty, L.H. Staib, and J.S. Duncan. Deformable boundary finding in medical images by integrating gradient and region information. IEEE Trans. Medical Imaging,
15(6):859-870, 1996.

[3] T.F. Chan and L.A. Vese. Active contours without edges. 
IEEE Trans. Image Proc., 10(2):266-277, 2001.

[4] T.F. Cootes and C.J. Taylor. Statistical models of appearance for medical image analysis and computer vision. In Proc. SPIE Medical Imaging, 2001.

[5] W. T. Freeman and J. B. Tenenbaum. Learning bilinear models for two-factor problems in vision. In Proceedings of IEEE Computer Vision and Pattern Recognition 1997, 1997.

[6] A. Hill, A. Thornham, and C.J. Taylor. Model-based interpretation of 3-D medical images. In Proceedings of 4th British Machine Vision Conference, pages 339-348, September 1993.

[7] S. Joshi. Large Deformation Diffeomorphisms and Gaussian Random Fields for Statistical Characterization of Brain Submanifolds. PhD thesis, Washington University, 1997.

[8] M. Kass, A. Witkin, and D. Terzopoulos. Snakes: active contour models. In Proc. Int. Conf. Computer Vision, London, June 1987.

[9] A. Kelemen, G. Szekely, and G. Gerig. Elastic modelbased segmentation of 3-D neuroradiological data sets. IEEE Transactions on Medical Imaging, 18(10):828-839, October 1999.

[10] Michael Leventon, Eric Grimson, and Olivier Faugeras. Statistical shape influence in geodesic active contours. In Proceedings of CVPR 2000, 2000.

[11] S. Osher and J.A. Sethian. Fronts propagating with curvature-dependent speed: Algorithms based on HamiltonJacobi formulation. J. Comput. Phys., 79:12-49, 1988.

[12] N. Paragios and R. Deriche. Geodesic active contours and level sets for the detection and tracking of moving objects. IEEE Trans. Pattern Anal. Machine Intell., 22(3):266-280, 2000.

[13] S. Pizer, G. Gerig, S. Joshi, and S. Aylward. Multiscale medial shape-based analysis of image objects. Proceedings of the IEEE, 91(10):1670-1679, 2003.

[14] S. Roweis and L. Saul. Nonlinear dimensionality reduction by locally linear embedding. Science, 290:2323-2326, 2000.

[15] Dinggang Shen and Christos Davatzikos. An adaptive-focus deformable model using statistical and geometric information. IEEE Transactions on Pattern Analysis and Machine Intelligence, 22(8):906-913, August 2000.

[16] Dinggang Shen, Yiqiang Zhan, and Christos Davatzikos. Segmentation of prostate boundaries from ultrasound images using statistical shape model. IEEE Transactions on Medical Imaging, 22(4), April 2003.

[17] M. Styner, G. Gerig, S. Pizer, and S. Joshi. Automatic and robust computation of 3D medial models incorporating object variability. International Journal of Computer Vision, 2002. To appear.

[18] J.B. Tenenbaum, V. de Silva, and J.C. Langford. A global geometric framework for nonlinear dimensionality reduction. Science, 290:2319-2323, 2000.
[19] A. Tsai, W. Wells, C. Tempany, E. Grimson, and A. Willsky. Coupled multi-shape model and mutual information for medical image segmentation. In IPMI 2003, pages 185-197, 2003.

[20] A. Tsai, A. Yezzi, W. Wells, C. Tempany, D. Tucker, A. Fan, E. Grimson, and A. Willsky. A shape based approach to curve evolution for segmentation of medical imagery. IEEE Trans. Medical Imaging, 22(2), February 2003.

[21] Greg Turk and James F. O'Brien. Shape transformation using variational implicit functions. In Computer Graphics (Proc. SIGGRAPH '99), pages 335-342, 1999.

[22] Y. Wang and L. H. Staib. Integrated approaches to nonrigid registration in medical images. In Proceedings of IEEE WACV 1998, pages 102-108, October 1998.
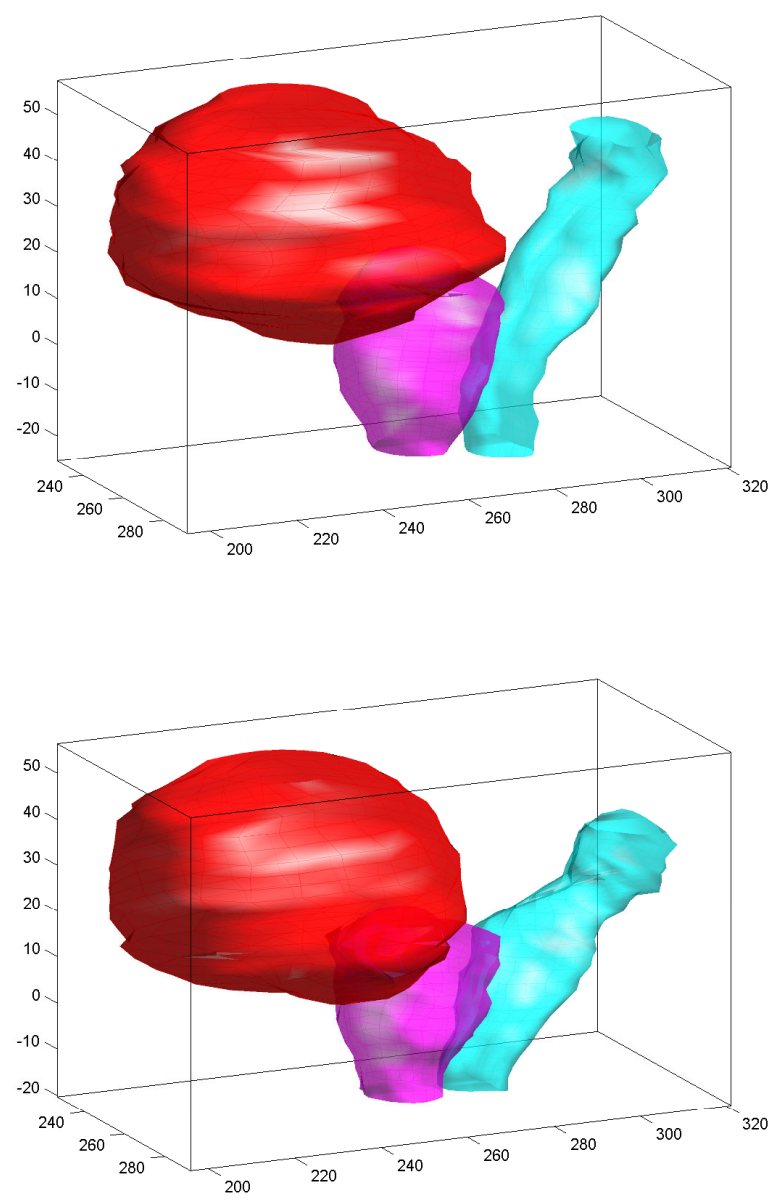

Figure 5: Segmentation results for patient 2664. Top: 3D segmentation result at convergence. Bottom: hand-drawn ground-truth contours supplied by a radiation physicist. 